Abstract Dark-field illumination is shown to make planar chiral nanoparticle arrangements exhibit circular dichroism in extinction, analogous to true chiral scatterers. Single oligomers, consisting rotationally symmetric arrangements of gold nanorods, are experimentally observed to exhibit circular dichrosim at their maximum scattering with strong agreement to numerical simulation. A dipole model is developed to show that this effect is caused by a difference in the projection of a nanorod onto the handed orientation of electric fields created by a circularly polarized dark-field normally incident on a glass-air interface. Owing to this geometric origin, the wavelength of the peak chiral response is experimentally shown to shift depending on the separation between nanoparticles. All presented oligomers have physical dimensions less than the operating wavelength, and the applicable extension to closely packed planar arrays of oligomers is demonstrated to amplify the magnitude of circular dichroism. This realization of strong chirality in these oligomers demonstrates a new path to engineer optical chirality from planar devices using dark-field illumination.

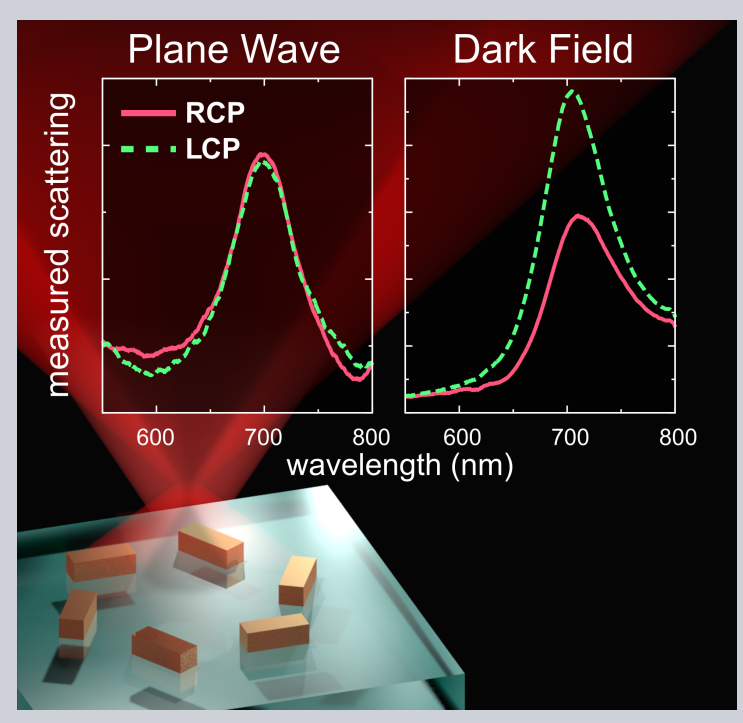

\title{
Optical chirality from dark-field illumination of planar plasmonic nanostructures
}

\author{
Yongsop Hwang ${ }^{1,2}$, Ben Hopkins ${ }^{3,4,}{ }^{*}$, Dapeng Wang ${ }^{1}$, Arnan Mitchell ${ }^{2}$, Timothy J. Davis ${ }^{5}$, \\ Jiao Lin ${ }^{1,2}$ and Xiao-Cong Yuan ${ }^{1, *}$
}

Dark-field (DF) imaging-illumination is one of the more straightforward avenues to directly observe resonances of single plasmonic nanoparticles, as used in though which it enables nanoparticle-assisted monitoring of few or single molecules [1-3] and, and even spatially resolved monitoring of reactions [4]. Yet DF is less common than bright-field in the related pursuit of discriminating between enantiomers of chiral molecules. The operation itself is existing mechanism for chiral discrimination nonetheless remains portable to DF settings: existing investigations have effectively investigations have sought to introduce a bias in the signed magnitude of local helicity density $h=$ $\operatorname{Im}\left\{\mathbf{E}^{*} \cdot \mathbf{H}\right\}$, to promote a net difference in coupling strength between a nanoparticle antenna and oppositely handed chiral molecules [5,6]. The nanoparticle geometry is therefore In this pursuit, the nanoparticle geometry represents a design freedom used to bias the helicity distribution generated under left-circular (LCP, positive $h$ ) or right-circular (RCP, negative $h$ ) polarized illuminations, for instance: it was. For instance, it has been shown that a nanoparticle antenna could foreseeably be designed to scatter only a single helic- ity of light [7]. However, the The majority of experimental investigations have, however, pursued an intermediary goal using true chiral geometries to produce circular dichroism (CD), : a variation in the extinction (total power dissipated) from LCP and RCP illuminations, and then relying which then relies on some degree of helicity conservation in scattering [8] to create athe helicity bias. Here we reveal that DF illumination is beneficial for this task because it: (i) enables simple planar chiral nanoparticle arrangements to exhibit $\mathrm{CD}$ in a manner comparable to true chiral scatterers, and (ii) allows employment of desireable geometric symmetries that would otherwise be expected to suppress CD.

In Figure 1, we show the experimental scattering spectra of for a planar chiral gold nanorod oligomer, depicted in (a), under RCP and LCP illuminations from: (c) normal incidence plane waves, and (d) DF with a 0.8-0.95 NA condenser. The DF scattering signal $S$ is measured as the transmission collected by a 0.6 NA objective lens, and this-which can be seen to vary significantly between RCP and LCP under DF illumination, whereas no variation. Conversely, no variation between RCP and LCP is seen in the extinction

\footnotetext{
${ }^{1}$ Nanophotonics Research Centre, Shenzhen University \& Key Laboratory of Optoelectronic Devices and Systems of Ministry of Education and Guangdong Province, College of Optoelectronic Engineering, Shenzhen University, Shenzhen 518060, China ${ }^{2}$ School of Engineering, RMIT University, Melbourne, VIC 3001, Australia ${ }^{3}$ School of Applied and Engineering Physics, Cornell University, Ithaca, NY 14853 , USA ${ }^{4}$ Nonlinear Physics Centre, Research School of Physics and Engineering, Australian National University, Canberra, ACT 2601, Australia ${ }^{5}$ School of Physics, University of Melbourne, VIC 3052, Australia

*Corresponding author: e-mail: ben.hopkins@anu.edu.au, e-mail: xcyuan@szu.edu.cn
}

This is the author manuscript accepted for publication and has undergone full peer review but has not been through the copyediting, typesetting, pagination and proofreading process, which may lead to differences between this version and the Version of Record. Please cite this article as doi: 10.1002/lpor.201700216 
a

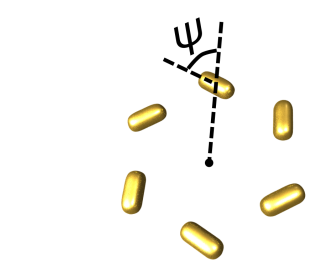

b

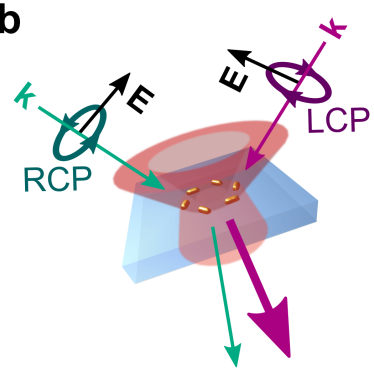

C

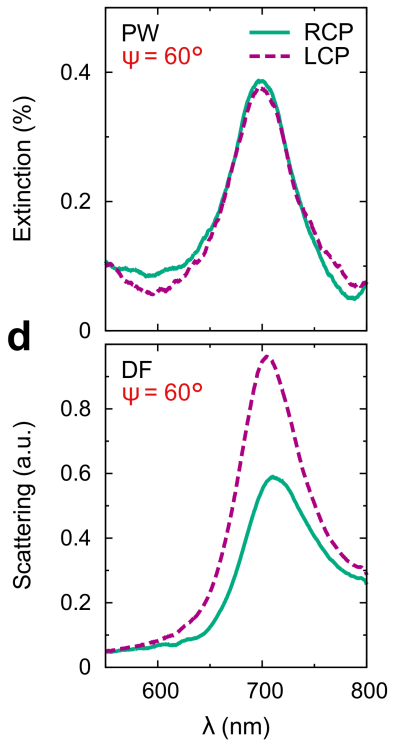

e

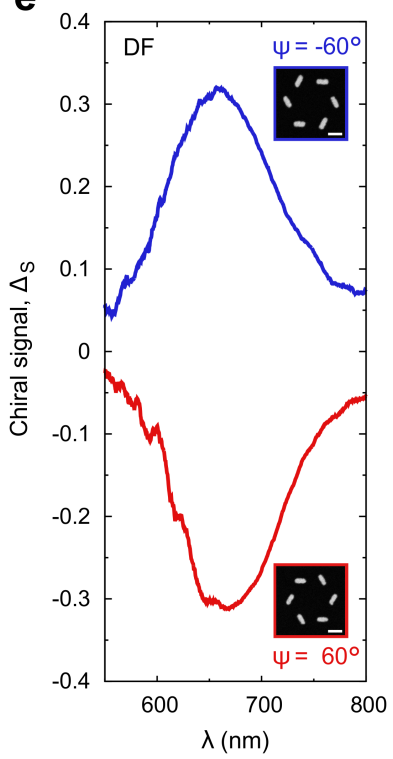

Figure 1 Schematics of the (a) planar gold nanorod oligomer and (b) dark-field illumination. The experimentally measured (c) extinction spectra for plane waves (PW) and (d) scattering spectra for dark-field (DF) are shown for circularly polarized incident light with nanorod orientation set to $\psi=60^{\circ}$. Results are also presented in terms of (e) chiral signal $\Delta_{S}=\left(S_{\mathrm{RCP}}-S_{\mathrm{LCP}}\right) /\left(S_{\mathrm{RCP}}+S_{\mathrm{LCP}}\right)$ for nanorod orientations $\psi=-60^{\circ}$ and $60^{\circ}$ from DF illumination. The dimensions of the nanorods were measured to be $35 \times 86 \mathrm{~nm}$, and the center-to-center distance between two opposing nanorods is $D=340 \mathrm{~nm}$. The scale bar in the insets is $100 \mathrm{~nm}$.

of generated under illumination by normal incidence plane waves, which was measured by subtracting the transmission from unity. The This observed absence of chiral signal under normal plane wave illumination will follow from is expected due to having 3-fold or more rotational symmetry when assuming each nanorod supports only a single dipole moment(see Supperting Information), as derived in $\S$ VI of the Supporting Information. The resonant peaks of $S$ under DF illumination in Figure $1 \mathrm{~d}$ are found at the wavelength of $704 \mathrm{~nm}$ and $713 \mathrm{~nm}$ for LCP and RCP, respectively. To quantify the chiral response, we define a chiral signal $\Delta_{S}=\left(S_{\mathrm{RCP}}-S_{\mathrm{LCP}}\right) /\left(S_{\mathrm{RCP}}+S_{\mathrm{LCP}}\right)$, as plotted in Figure 1e. The single gold nanorod oligomer exhibits over $\Delta_{S}=0.3$, and does so on resonance, which makes it comparable to geometrically chiral antennas $[9,10]$, or obliquely incident plane waves on planar antennas that lack inversion symmetry [11]. Notably, the oligomer has inversion symmetry in the absence of substrate, meaning CD is not expected for any obliquely or normally incident oblique or normal incidence plane wave (see $\S$ IV of the Supporting Information). The oligomer also has 6-fold rotational symmetry, which is an example of desireable symmetry: it forbids scattering of oppositely handed fields in the transmission direction along its principle axis [12], thereby promoting the generation of a uniform helicity distribution under circularly polarized illumination. The chiral signal in-In the coming paragraph, we will derive the origin of the observed chiral signal for the considered oligomers using a dipole model. This model concludes that the observed signal is due to true circular dichroism in extinction. As circular dichroism in extinction is usually restricted to three-dimensional chiral nanostructures with normal incidence, the magnitude of chiral signal in Figure 1d,e is therefore largely unexpected, yet it is promising for the broad pursuit of chiral optical response in . The result is subsequently very relevant for two-dimensional plasmonic structures that seek to avoid the challenges involved with fabrieating true three-dimensional chirality [13-18]. However, as presence of circular dichroism is largely unexpected, we will now derive its existence in such oligomers rigorously. planar chiral nanostructures, which have predominately been utilized instead for circular conversion dichroism, or (to a lesser extent) circular dichroism in absorption [13-21].

Let us assume each gold nanorod behaves as an anisotropic point dipole aligned to the long-axis of the nanorod, and denote this orientation with a unit vector $\hat{\boldsymbol{\psi}}$. Given the propagation axis of the circularly polarized DF illumination is parallel to the rotational symmetry axis of the oligomer, a symmetric rotation of the global coordinate system is equivalent to a phase shift of the applied field. The amplitude of each nanorod's dipole moment is therefore equal, up to a phase shift, with that of every other nanorod, making it sufficient to restrict our consideration to a single nanorod. So let us define a nanorod's dipole moment at a distance $\delta_{x}$ along the $\hat{\mathbf{x}}$-axis, with the substrate a distance $\delta_{z}$ below $(-\hat{\mathbf{z}})$ the dipole. A basic DF illumination can be defined as an annular ring of circularly polarized plane waves with incoming wavevectors $\mathbf{k}$ forming a circle in the tranverse $\mathbf{k}$-space with polar incident angle $\phi$, as illustrated in Figure 2(a). Our dipole will interact with the applied electric field projected onto its orientation $\hat{\boldsymbol{\psi}}$, which can be decomposed into the contributions from azimuthal, or transverse electric the azimuthal and the polar components of polarization, being the transverse electric (TE) component $\mathbf{E}_{\mathrm{TE}} \cdot \hat{\boldsymbol{\theta}} \cdot \hat{\boldsymbol{\psi}} \equiv \mathrm{E}_{\mathrm{TE}}$, 
a

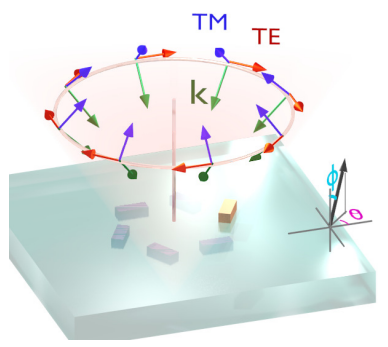

b

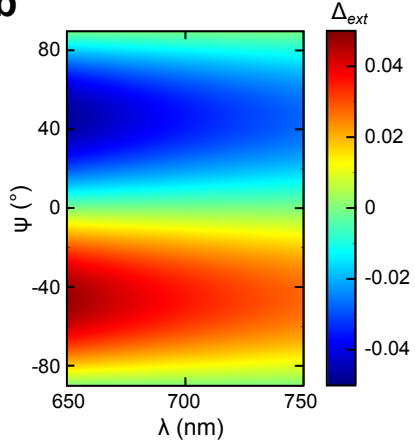

Figure 2 (a) Illustration of the annular ring of plane waves used in the dipole model Eq. 1 and 2, showing the TE and TM components of electric field polarization. In (b) we show the predicted circular dichroism for extinction $\sigma$, expressed as a chiral signal $\Delta_{\text {ext }}=\left(\sigma_{\mathrm{RCP}}-\sigma_{\mathrm{LCP}}\right) /\left(\sigma_{\mathrm{RCP}}+\sigma_{\mathrm{LCP}}\right)$ from the angular distributions of plane waves in (a), modelling a dark-field illumination. This was calculated using Eq. 1 and 2, with $\delta_{x}=170 \mathrm{~nm}$ and $\delta_{z}=15 \mathrm{~nm}$.

polarizations and from polar, transverse magnetic and the transverse magnetic (TM) component $\mathbf{E}_{\mathrm{TM}} \cdot \hat{\boldsymbol{\phi}} \cdot \hat{\boldsymbol{\psi}} \equiv \mathrm{E}_{\mathrm{TM}}$, pelarizations. The - Note that the TM field includes a phase factor of $\pm i$ relative to TE, to impose the circular polarization. The resulting projection , of of the total electric field onto our dipole, oriented dipole will then be given by: $\mathrm{E}^{( \pm)}=\mathrm{E}_{\mathrm{TE}}^{( \pm)}+\mathrm{E}_{\mathrm{TM}}^{( \pm)}$, with \pm indicating LCP and RCP, respectively. We provide derivation of $\mathrm{E}_{\mathrm{TE}}^{( \pm)}$and $\mathrm{E}_{\mathrm{TM}}^{( \pm)}$in $\S$ II of the Supporting Information, and state the result-:

$$
\begin{aligned}
\mathrm{E}_{\mathrm{TE}}^{( \pm)}= & \pm i \pi\left(J_{0}\left(-k \delta_{x} \sin \phi\right) e^{\mp i \psi}+J_{2}\left(-k \delta_{x} \sin \phi\right) e^{ \pm i \psi}\right) \\
& \cdot\left(1+r_{\mathrm{TE}} e^{2 i k \delta_{z} \cos \phi}\right) \\
\mathrm{E}_{\mathrm{TM}}^{( \pm)}= & \pm i \pi\left(J_{0}\left(-k \delta_{x} \sin \phi\right) e^{\mp i \psi}-J_{2}\left(-k \delta_{x} \sin \phi\right) e^{ \pm i \psi}\right) \\
& \cdot \cos \phi \cdot\left(1-r_{\mathrm{TM}} e^{2 i k \delta_{z} \cos \phi}\right)
\end{aligned}
$$

Here $r_{\mathrm{TE}}$ and $r_{\mathrm{TM}}$ are Fresnel reflection coefficients for the air-substrate interface, and $J_{n}$ is the $n^{\text {th }}$ order Bessel function of the first kind. A chiral signal can next be defined for the magnitude of the applied electric field projected onto $\hat{\boldsymbol{\psi}}$, being: $\Delta_{\mathrm{E}}=\left(\left|\mathrm{E}^{(-)}\right|^{2}-\left|\mathrm{E}^{(+)}\right|^{2}\right) /\left(\left|\mathrm{E}^{(-)}\right|^{2}+\left|\mathrm{E}^{(+)}\right|^{2}\right)$. When using our dipole model, $\Delta_{\mathrm{E}}$ is equal to the chiral signal for the extinction $\sigma$ of the whole oligomer $\Delta_{\text {ext }}=\left(\sigma_{\mathrm{RCP}}-\sigma_{\mathrm{LCP}}\right) /\left(\sigma_{\mathrm{RCP}}+\sigma_{\mathrm{LCP}}\right)$, see $\S \mathrm{VI}$ of the Supporting Information. Cireular As such, circular dichroism only exists here in the considered oligomer due to a difference in the magnitude of the electric field projected onto $\hat{\boldsymbol{\psi}}$. Notably, when there is no substrate $\mathrm{E}^{( \pm)}=-\left(\mathrm{E}^{(\mp)}\right)^{*}$, hence $\left|\mathrm{E}^{(+)}\right|^{2}=\left|\mathrm{E}^{(-)}\right|^{2}$, and $\Delta_{\mathrm{E}}$ is precisely zero. In Figure 2(b) we account, we account also for the substrate and integrate $\mathrm{E}^{( \pm)}$over $\phi$ when assuming uniform amplitude plane waves are distributed on an annular solid angle section in $\mathbf{k}$-space $(\mathrm{d} \Omega=\sin \phi d \theta \mathrm{d} \phi)$ truncated to the experimental DF condenser's $0.8-0.95$ NA range. ${ }^{1}$ Under these conditionswe see,$\Delta_{\mathrm{E}}$ is nonzero, noting that $\Delta_{\mathrm{E}}$ remains zero for normal incidence plane waves, even with the substrate. To understand the role of the DF, its electric field within the plane of the nanorods will have but $\Delta_{\mathrm{E}}$ is nonzero for DF illumination. This can be understood as a consequence of the applied electric field from the DF having an elliptical polarization at locations off the propagation axis $\left(\delta_{x} \neq 0\right)$, leading within the plane of the nanorods. Before considering circular dichroism, we recognize that this ellipticity will lead to increased extinction when polarization major axis aligns with nanorod orientation $\hat{\boldsymbol{\psi}}$. Furthermore, Regarding circular dichroism, we note that transforming $\psi \rightarrow-\psi$ in Eq. 1 and 2 is equivalent to $\left|\mathrm{E}^{(+)}\right|^{2} \rightarrow\left|\mathrm{E}^{(-)}\right|^{2}$, meaning which means circular dichroism exists whenever the magnitude of electric field projected onto $\psi$ is not equal to that projected onto $-\psi$. Given $\psi$ dependence cannot be separated as a common unitary factor from the sum of $\mathrm{E}_{\mathrm{TE}}^{( \pm)}$and $\mathrm{E}_{\mathrm{TM}}^{( \pm)}$, as defined in (1) and (2); circular dichroism can be expected whenever the applied electric field's major polarization axis is aligned with any $\psi \neq 0,90^{\circ}$, where 0 and $90^{\circ}$ are angles for which $e^{ \pm i \psi}=-e^{\mp i \psi}$. Without a-Now, when neglecting the substrate $\left(r_{\mathrm{TE}}=r_{\mathrm{TM}}=0\right)$, and also given the Bessel functions in (1) and (2) are real valued, then: $\left|\mathrm{E}_{\mathrm{TE}}^{( \pm)}\right|$is maximized at $\psi=0$, and $\left|\mathrm{E}_{\mathrm{TM}}^{( \pm)}\right|$is maximized at $\psi=90^{\circ}$, where these two maxima are $\frac{\pi}{2}$ out of phase. In other words, the major polarization axis is aligned to either $\psi=0$ or $90^{\circ}$ (whichever has larger amplitude), and hence no circular dichroism. With However, with a substrate, the phase acquired in reflection due to $\delta_{z}$ makes the relative phase of $\mathrm{E}_{\mathrm{TE}}^{( \pm)}$and $\mathrm{E}_{\mathrm{TM}}^{( \pm)}$depend on $\phi$. The in-phase components of $\mathrm{E}_{\mathrm{TE}}^{( \pm)}$and $\mathrm{E}_{\mathrm{TM}}^{( \pm)}$when integrating over $\phi$ are therefore no longer going to uniformly be at $\psi=0$ or $90^{\circ}$, indicating. This indicates the major polarization axis will be rotated, thereby allowing and thereby allows circular dichroism. It is

It is also worth recognizing that this the radial component of ellipticity we predict in the periphery of a beam resembles that the DF resembles that generated from the optical spin-Hall effect [22] at an interface. $A$-a dielectric interface. More specifically, a linear-polarized Gaussian incident on an interface is known to generate a symmetric eross-polarizationcross-polarized component in the portion of the beam periphery perpendicular to the original polarization axis [23]. For a circular-polarized Gaussian, as the sum of two linear-polarized Gaussians, this same effect can be expected to rotate the major axis of the transverse elliptical polarization out of alignment with $\psi=0,90^{\circ}$, which is also what our model predicted. In stmmary, To summarize the analysis to now: ellipticity in the electric field distribution from a circularly polarized DF incident on a substrate can be

1 In $\S$ II of the Supporting Information, we also calculate $\Delta_{E}$ due to a DF modeled as a superposition of two Gaussians with NA 0.95 and NA 0.8 , which is used. This corresponds to what we use for the simulations in numeriesFigures 3 and 4, and show this predicts a circular dichroism in the dipole model with-whose magnitude is closer to that seen in experimentthe presented experiments. 
expected to provide preferential coupling of LCP and RCP into the considered oligomers, and have an. This ellipticity and circular dichroism will moreover be observable by the corresponding angular dependence on the nanorod orientation $\psi$.

a

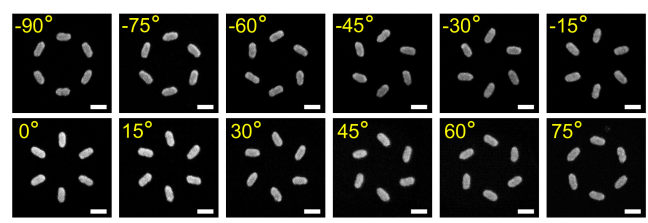

b

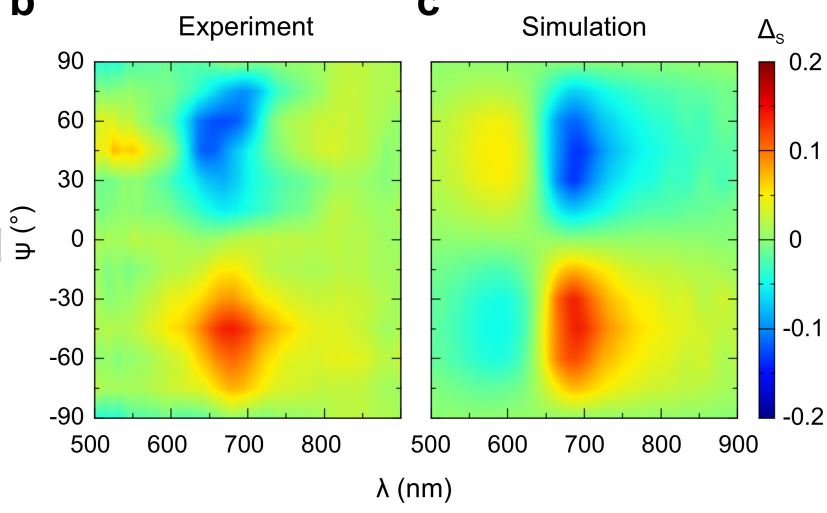

Figure 3 The variation of the observed chiral response of the plasmonic hexamers depending on the orientation angle $\psi$ of the gold nanorods. (a) SEM images of the plasmonic hexamers with different $\psi$, the scale bar is $100 \mathrm{~nm}$. (b) Experimental measurements and (c) numerical simulations of the chiral signal $\Delta_{S}$ as functions of nanorod orientation $\psi$.

We now show this angular dependence experimentally. Gold nanorod oligomers were fabricated for the full $180^{\circ}$ range of possible $\psi$ in $15^{\circ}$ steps, as shown in Figure $3 \mathrm{a}$. The dimensions of the fabricated nanorods were measured to be $43 \times 94 \mathrm{~nm}$. The measured chiral signal $\Delta_{S}$ from experiment and from simulation using CST Microwave Studio, produce the maps presented in Figure $3 \mathrm{~b}$ and $\mathrm{c}$, respectively. Both show the $\psi$-dependent circular dichroism that we expected from the dipole model: no chiral response is observed at $\psi=0,90^{\circ}$, and opposite sign of chiral signal observed for oppositely handed chiral oligomers. The peak chiral signal here is also occurring in the vicinity of the oligomer's resonant frequency, which is expected because this is necessary to ensure large anisotropy of the nanobars. The measured chiral signal is thereby also associated with significant differential scattering signal, rather than suppressing the denominator of $\Delta_{S}$, and this has allowed measuring strong chiral signal from even a single oligomer. The difference in seattering magnitude of several oligomers is shown later in Figure $4 \mathrm{a}$, and in simulationwe also-From simulation, we also expect to see a qualitative change in the local scattered electric field intensity as depicted in Figure 4c. There is now
We can now explore a second degree of freedom we have over that impacts the chiral signal: the transverse displacement of nanorods $\delta_{x}$. Referring to our model in EqEqs. 1 and 2 , if the nanorods optically are thin $\delta_{z} \approx 0$, we assume the nanorods are optically thin $\left(k \delta_{z} \approx 0\right)$ and then the wavelength dependence of the applied field projection is entirely due to the size of determined entirely by the quantity $k \delta_{x}$, where $\delta_{x}$ relative to the wavelength fis the transverse displacement of nanoparticles relative to their collective origin In other words, the peak chiral signal should occur at a fixed value of $k \delta_{x}$ ). We should therefore expect the transverse displacement of nanorods to shift the . We can therefore expect that increasing $\delta_{x}$ will red-shift the peak chiral signal, without having to change changing the nanorod dimensions. In Figure 4, we present an experimental investigation of nanorod oligomers when varying the center-to-center distance between opposing nanorods $D=2 \delta_{x}$ from $260 \mathrm{~nm}$ to $420 \mathrm{~nm}$ in increments of $40 \mathrm{~nm}$. The corresponding scattering and chirality spectra are provided in Figure 4a and b, respectively. We do indeed observe a red-shift with increasing $D$ in the peak chiral signal from $\lambda=610 \mathrm{~nm}$ to $700 \mathrm{~nm}$, while the resonance wavelength of scattering signal remains largely stationary. The observed red-shift is understood from the dipole model having the wavelength dependence entirely on $k \delta_{x}$ which describes the spectral shifts to the longer wavelength as increasing the in-plane separation of the nanorods. The detailed calculation based on the model for different $\delta_{x}$ is given in Figure S1 in Supporting Information. This thereby provides an effective way to engineer the wavelength of chiral DF scattering from a subwavelength planar plasmonic structure, without changing the physical dimension of the consisting metal nanorods, such as if constrained by fabrication or assembly procedure.

The resonant frequency of scattering in Figure 4 is relatively constant, despite the changes to the oligomer dimensions, which suggests that we are observing a resonance that is dominantly determined by that of a single nanorod. The presented oligomers also have a subwavelength physical footprint, courtesy of quasistatic surface plasmon resonances in highly subwavelength photonic devices [24-28]. These two points suggest that we should be able to directly translate the chiral operation of a single oligomer into a homogenized metasurface platform. So we can explore arrays of oligomers, as to show relevance of these concepts also toward pursuit of plasmonic metasurfaces for polarization control, and other applications for flat optics [29-32] that capitalize on 2-dimensional fabrication. The single oligomer experiments in Figures 1, 3 and 4 have shown that each oligomer scatters light significantly more efficiently for either RCP or LCP, and these differences in scattering can generally be expected to affect the radiative coupling strength between an oligomer and its neighbors in the surrounding lattice. Moreover, by varying the spacing between the oligomers in the lattice, we are able to tune the phase and coupling strength between neighboring oligomers. In Figure 5, we present investigate this interplay through experimental measurements of arrays of nanorod oligomers with lattice periods ranging from $510 \mathrm{~nm}$ to 
a

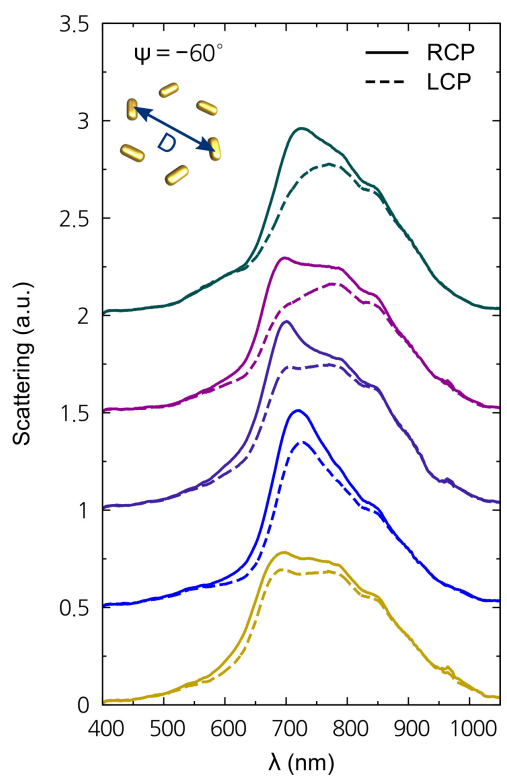

b

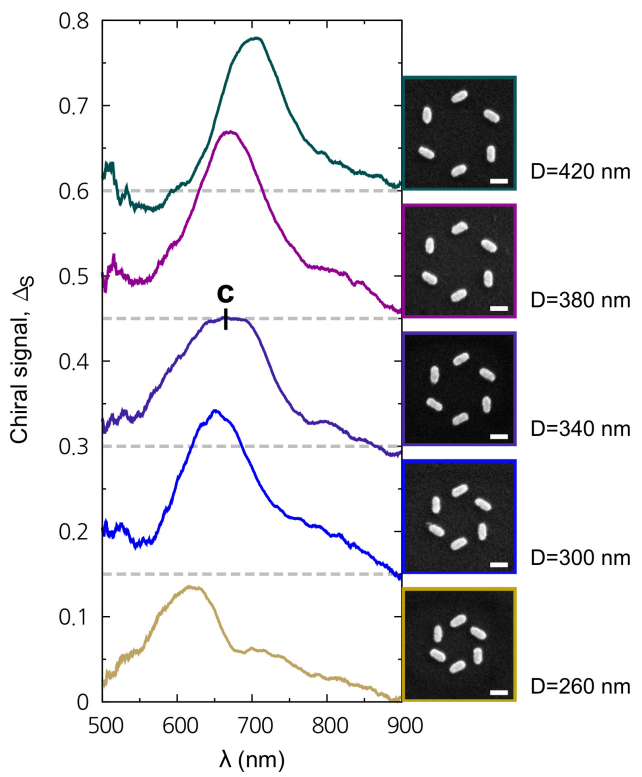

C

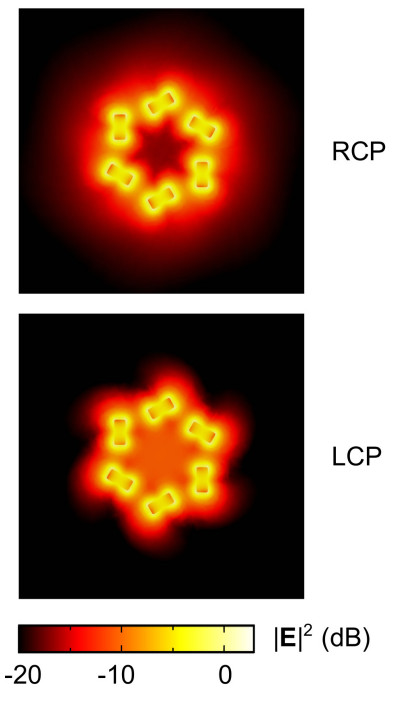

Figure 4 The measured (a) scattering and (b) chiral signal spectra for different separations $D$ between opposing nanorods. The SEM images have a scale bar of $100 \mathrm{~nm}$. Shown in (c) are simulated near-field profiles of $|\mathbf{E}|^{2}$ in $\mathrm{dB}$ under RCP and LCP illumination, for the case where $D=340 \mathrm{~nm}$ and $\lambda=660 \mathrm{~nm}$.

$1020 \mathrm{~nm}$. The strongest chiral response is observed for the array with $p=680 \mathrm{~nm}$ whose maximum chiral signal $\Delta_{S}$ is over 0.5 , approximately three times greater than the equivalent single oligomer in Figure 3. Yet, simultaneously, Additionally, no substantial wavelength shift is observed in the chiral signal peak, suggesting that the circular dichroism remains as the same field projection mechansim that occurs for the same underlying field projection mechanism exists as was the case for circular dichroism in the single oligomer. As streh, the The lattice period is thereby a parameter with which we can optimize the chiral signal magnitude without impacting wavelength shifts (Figure 5), and the nanoparticle spacing within a constituent oligomer is a parameter than governs wavelength shifts of the chiral signal (Figure 4).

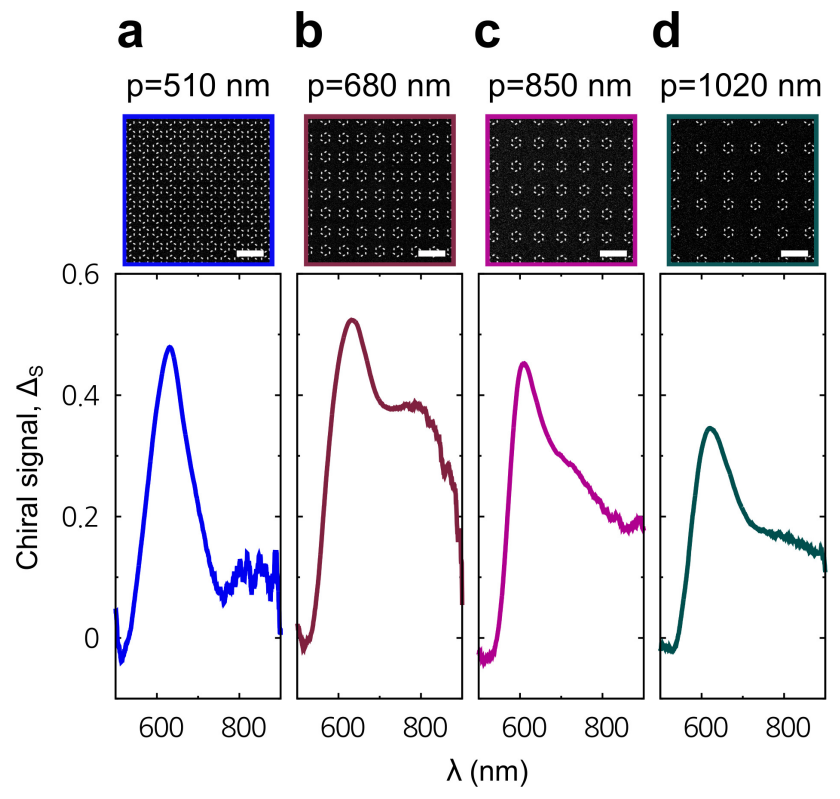

Figure 5 Chiral signal spectra of the arrays with the periods of (a) $510 \mathrm{~nm}$, (b) $640 \mathrm{~nm} 680 \mathrm{~nm},($ c) $850 \mathrm{~nm}$, and (d) $1020 \mathrm{~nm} . \psi$ and $D$ are fixed to be $-60^{\circ}$ and $340 \mathrm{~nm}$, respectively. The scale bar in the SEM images is $1 \mu \mathrm{m}$.

To conclude, we have realized a subwavelength scale planar device that exhibits strong optical chirality using arrangements of plasmonic nanoparticles illuminated by dark-field. Theoretical analysis using a dipole model has explained the origin of the chiral response is a difference in the geometric projection of a nanorod onto the handed 
orientation of electric fields created by a circularly polarized dark-field incident on a glass substrate. The dependence of the orientation angle of the nanorods has been systematically demonstrated with experiments and numerical simulations. It was also found that the wavelength range of the chiral response can be tuned by changing the distance between nanorods, and the magnitude can be amplified through the extension to closely packed, planar arrays of oligomers. Planar plasmonic chiral devices based on the demonstrated structures can therefore be employed in the place of true chiral geometries while also benefiting from simultaneous access to desireable geometric symmetries, including rotational symmetry considered here. These results foreseeably benefit pursuits that require preferential generation of left- and right-handed helicity fields, such as nanoparticleassisted discrimination of enantiomers.

Acknowledgements. The work is supported by the leading talents of Guangdong province program No. 00201505 and the Natural Science Foundation of Guangdong Province under No. 2016A030312010. This work was performed in part at the Melbourne Centre for Nanofabrication (MCN) in the Victorian Node of the Australian National Fabrication Facility (ANFF). BH acknowledges the discussions on theory and simulation with A.E. Miroshnichenko, Z. Fan, S. Trendafilov and M.R. Shcherbakov, the advice of F. Demming and C. Kremers (CST) on simulations using CST Microwave Studio, and the support from A.E. Miroshnichenko, Y.S. Kivshar and G. Shvets.

Key words: Plasmonics, Chirality, Subwavelength optics, Flat optics, Dark-field scattering

\section{References}

[1] A. D. McFarland and R.P. V. Duyne, Nano Lett. 3, 10571062 (2003).

[2] I. Ament, J. Prasad, A. Henkel, S. Schmachtel, and C. Sönnichsen, Nano Lett. 12, 1092-1095 (2012).

[3] M. C. Estevez, M. A. Otte, B. Sepulveda, and L.M. Lechuga, Anal. Chim. Acta 806, 55-73 (2014).

[4] M. A. Beuwer, M. W. J. Prins, and P. Zijlstra, Nano Lett. 15, 3507-3511 (2015).

[5] Y. Tang and A. E. Cohen, Physical Review Letters 104(16), 163901 (2010).

[6] S. Yoo and Q. H. Park, Phys. Rev. Lett. 114, 203003 (2015).

[7] I. Fernandez-Corbaton, M. Fruhnert, and C. Rockstuhl, Phys. Rev. X 6, 031013 (2016).

[8] M. Nieto-Vesperinas, Phys. Rev. A 92, 023813 (2015).

[9] M. Decker, M. Ruther, C. E. Kriegler, J. Zhou, C. M. Soukoulis, S. Linden, and M. Wegener, Opt. Lett. 34(16), 25012503 (2009).

[10] A. Kuzyk, R. Schreiber, Z. Fan, G. Pardatscher, E. M. Roller, A. Högele, F. C. Simmel, A. O. Govorov, and T. Liedl, Nature 483, 311314 (2012).

[11] I. Sersic, M. A. van de Haar, F. B. Arango, and A. F. Koenderink, Phys. Rev. Lett. 108, 223903 (2012).

[12] I. Fernandez-Corbaton, Opt. Express 21(24), 29885 (2013).

[13] V. A. Fedotov, P. L. Mladyonov, S. L. Prosvirnin, A. V. Rogacheva, Y. Chen, and N. I. Zheludev, Phys. Rev. Lett. 97, 167401 (2006).
[14] F. Eftekhari and T. Davis, Physical Review B 86(7), 075428 (2012).

[15] L. Du, S. S. Kou, E. Balaur, J. J. Cadusch, A. Roberts, B. Abbey, X. C. Yuan, D. Tang, and J. Lin, Nature Communications 6, 10051 (2015).

[16] B. Hopkins, A. N. Poddubny, A. E. Miroshnichenko, and Y. S. Kivshar, Laser \& Photonics Reviews 10(1), 137-146 (2016).

[17] S. Zu, Y. Bao, and Z. Fang, Nanoscale 8(7), 3900-3905 (2016).

[18] A. B. Khanikaev, N. Arju, Z. Fan, D. Purtseladze, F. Lu, J. Lee, P. Sarriugarte, M. Schnell, R. Hillenbrand, M. A. Belkin, and G. Shvets, Nature Communications 7, 12045 (2016)

[19] V. Fedotov, A. Schwanecke, N. Zheludev, V. Khardikov, and S. Prosvirnin, Nano Letters 7(7), 1996-1999 (2007).

[20] Z. Wang, Y. Wang, G. Adamo, B. H. Teh, Q. Y.S. Wu, J. Teng, and H. Sun, Advanced Optical Materials 4(6), 883888 (2016).

[21] Z. Wang, B. H. Teh, Y. Wang, G. Adamo, J. Teng, and H. Sun, Applied Physics Letters 110(22), 221108 (2017).

[22] K. Y. Bliokh, F. J. Rodriguez-Fortuno, F. Nori, and A. V. Zayats, Nat. Photon. 9, 796-808 (2015).

[23] K. Y. Bliokh and Y. P. Bliokh, Phys. Rev. Lett. 96, 073903 (2006)

[24] W. L. Barnes, A. Dereux, and T. W. Ebbesen, Nature 424(6950), 824-830 (2003).

[25] S. H. Kwon, J. H. Kang, C. Seassal, S. K. Kim, P. Regreny, Y. H. Lee, C. M. Lieber, and H. G. Park, Nano Letters 10(9), 3679-3683 (2010).

[26] J. H. Kang, K. Kim, H. S. Ee, Y. H. Lee, T. Y. Yoon, M. K. Seo, and H. G. Park, Nature Communications 2, 582 (2011).

[27] J. Lin, J. B. Mueller, Q. Wang, G. Yuan, N. Antoniou, X. C. Yuan, and F. Capasso, Science 340(6130), 331-334 (2013).

[28] D. E. Gómez, Y. Hwang, J. Lin, T. J. Davis, and A. Roberts, ACS Photonics 4(7), 1607-1614 (2017).

[29] N. Yu and F. Capasso, Nature Materials 13(2), 139-150 (2014).

[30] G. Zheng, H. Mühlenbernd, M. Kenney, G. Li, T. Zentgraf, and S. Zhang, Nature Nanotechnology 10(4), 308-312 (2015).

[31] Y. Hwang and T. J. Davis, Applied Physics Letters 109(18) (2016).

[32] H. S. Ee and R. Agarwal, Nano letters 16(4), 2818-2823 (2016). 


\section{University Library}

\section{- M M N E R VA A gateway to Melbourne's research publications}

Minerva Access is the Institutional Repository of The University of Melbourne

\section{Author/s:}

Hwang, Y;Hopkins, B;Wang, D;Mitchell, A;Davis, TJ;Lin, J;Yuan, X-C

Title:

Optical Chirality from Dark-Field Illumination of Planar Plasmonic Nanostructures

Date:

2017-11-01

Citation:

Hwang, Y., Hopkins, B., Wang, D., Mitchell, A., Davis, T. J., Lin, J. \& Yuan, X. -C. (2017).

Optical Chirality from Dark-Field Illumination of Planar Plasmonic Nanostructures. LASER \& PHOTONICS REVIEWS, 11 (6), https://doi.org/10.1002//por.201700216.

Persistent Link:

http://hdl.handle.net/11343/293576 